\title{
The role of imaging in the evaluation of extraskeletal Ewing's sarcoma
}

\section{P Barnardt, F Roux}

Division of Clinical Oncology, Department of Medical Imaging and Clinical Oncology, Stellenbosch University

P Barnardt, MB ChB, Dip Onc

Division of Radiology, Department of Medical Imaging and Clinical Oncology, Stellenbosch University

F Roux, MB ChB, DA (SA)

Corresponding author: P Barnardt (pieterb@sun.ac.za)

Primary bone tumours account for $5 \%$ of all adolescent and child cancers. When Ewing's sarcoma arises in soft tissue rather than bone, it is referred to as extraskeletal Ewing's sarcoma (ESS). Computed tomography (CT) scanning and magnetic resonance imaging (MRI) features of Ewing's sarcoma are non-specific, and a radiological differential diagnosis should be considered. Ewing's sarcoma is confirmed by features on histological analysis. In young people who present with soft-tissue tumours, ESS should be considered. In the management of patients with tumours, imaging techniques are useful for biopsy guidance, evaluating the possibility of resection, and tumour response to treatment.

S Afr J Rad 2013;17(1):30-33. DOI:10.7196/SAJR.790

Primary bone tumours account for $5 \%$ of all adolescent and child cancers. The Ewing's sarcoma family of tumours is an aggressive form of childhood cancer and comprises the second most common primary bone tumour that includes Ewing's sarcoma, Askin's tumour and peripheral primitive neuro-ectodermal tumour (PNET). When Ewing's sarcoma arises in soft tissue (25\%) rather than bone, it is referred to as extraskeletal Ewing's sarcoma (EES). Before the introduction of chemotherapy, only $10 \%$ of patients survived but, with current management, $75 \%$ with localised disease will survive. ${ }^{[1,2]}$ We describe the presentation and outcome of 2 cases of EES.

\section{Case 1}

A 17-year-old boy presented with chronic lower backache for 6 months. The pain was located in the lower lumbar region and associated with right-sided L3 radiculopathy. On physical examination, a right paravertebral swelling was noted. The neurological examination was intact, with no loss of sphincter control. Special investigations included normal blood work and chest X-ray (CXR). Lumbar spine X-ray confirmed a subtle sclerotic change, isolated to the right pedicle of L3 (Fig. 1). A ${ }^{99 m}$ TcMDP whole-body bone scintigraphy study reported abnormal osteoblastic activity in L2/L3 not typical of Ewing's sarcoma.

Computed tomography (CT) and magnetic resonance imaging (MRI) were performed. The selected axial CT slice at level L3 noted sclerosis and smooth volumetric expansion of the right pedicle. These bony changes were not in keeping with a primary bony neoplasm and thought to be reactive change secondary to the large associated soft-tissue tumour (Fig. 2). Numerous small pulmonary nodules in the peripheral lung zones of the lower lobes suggested pulmonary metastases.

A MRI gadolinium-enhanced T1-weighted fat-saturated axial slice at L2/L3 level showed a lobulated avidly enhancing right paraspinal soft-

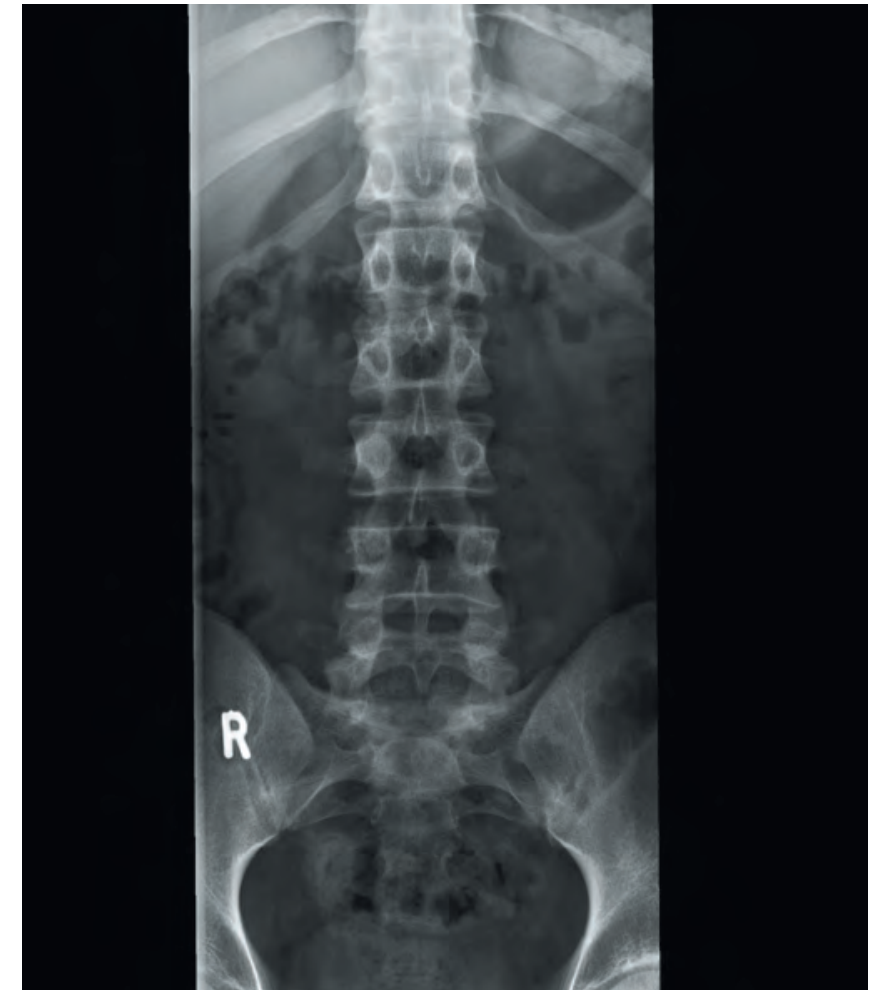

Fig. 1. AP lumbar radiograph. Note the subtle isolated sclerotic change to the right L3 pedicle.

tissue mass displacing and invading the psoas muscle and erector spinae muscle group and extended to insinuate into the spinal canal, displacing and compressing the thecal sac to the left (Fig. 3). 


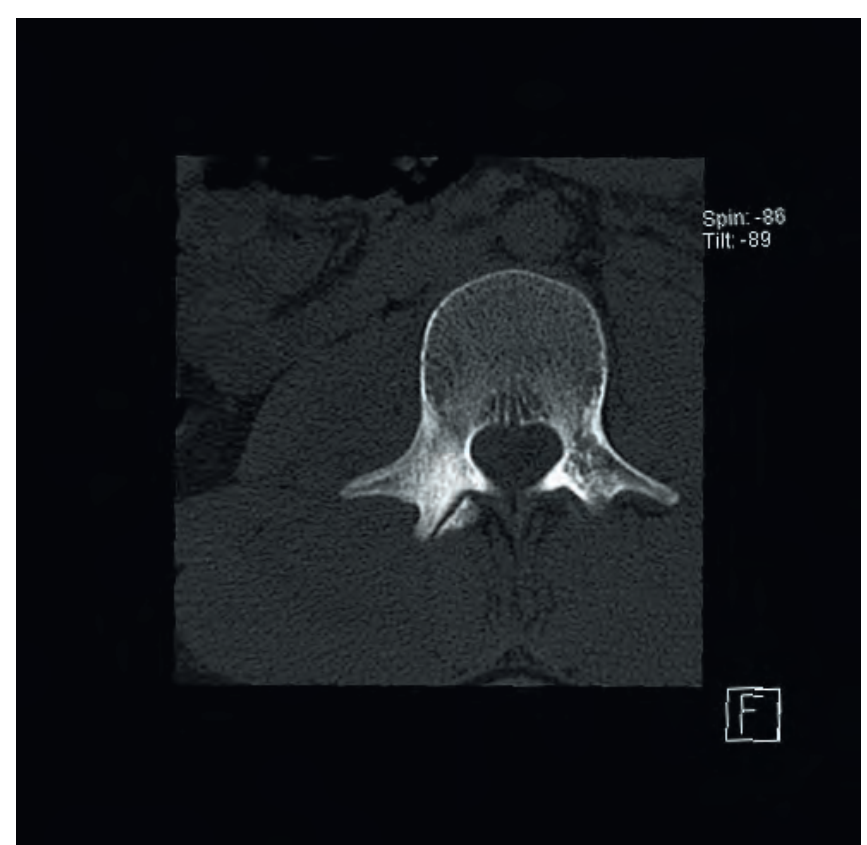

Fig. 2. A selected L3 axial CT slice (bony window). Note the sclerosis and smooth volumetric expansion of the the right pedicle.

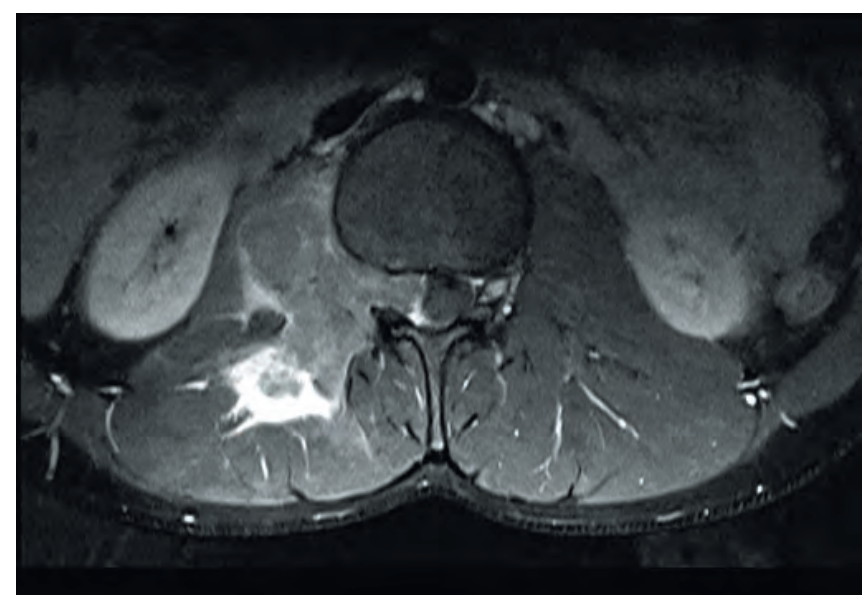

Fig. 3. A gadolinium-enhanced T1-weighted fat-saturated axial slice at L2/ L3 level showing an avidly lobulated enhancing right paraspinal soft-tissue mass displacing and invading the psoas and erector spinae muscle group and extended into the spinal canal, displacing and compressing the thecal sac to the left.

Histology revealed a solidly packed, lobular pattern of uniform small round malignant blue cells. The individual cells had round-to-ovoid nuclei and fine powdery chromatin with small nucleoli. The cytoplasm was scant and vacuolated. Immunohistochemistry stained strongly positive for the tumour membranous cells (CD99). Based on the available evidence, a PNET/Ewing's sarcoma was the most likely diagnosis.

At a multidisciplinary team meeting, a clinico-pathological and radiological correlation of the MRI and CT confirmed the large paraspinal mass of the right L3 pedicle and transverse process with soft-tissue mass in the extradural space. These findings were consistent with a malignant tumour and suggested PNET/Ewing's sarcoma. According to the American Joint Committee on Cancer (AJCC) classification system, the patient was staged as a G3T2N0M1, Stage IV Ewing's sarcoma.
Systemic multi-agent chemotherapy consisted of VAIA (vincristine, doxorubicin alternated with actinomycin-D and ifosphamide) every 4 weeks. He completed 6 cycles, and restaging investigations included a post-treatment MRI that reported anterior end plate irregularity at L2/L3 level with herniation of disc material within the vertebral body. There was loss of disc height at L1 and L2 and a small area of ill-defined enhancement related to the right pedicle of the L3 vertebral segment. No obvious overt enhancing tumour with volumetric gain was visualised on the MRI examination. A post-chemotherapy chest CT confirmed complete resolution of the lung metastases. The patient completed definitive external beam radiotherapy that included the gross tumour volume with a $2 \mathrm{~cm}$ margin (45 Gray in 1.8 fractions) but no boost owing to the spinal cord dose limitation. This was followed by 6 months of maintenance chemotherapy for a total of 12 cycles. He is currently in complete clinico-radiological remission and disease-free for 24 months and continues with a normal active life.

\section{Case 2}

A 22-year-old man presented with a history of intermittent but progressive pain located in the pelvic region and associated left leg weakness for 8 months. Physical examination revealed chronic illness with localised pain to the left pubic rami. Special investigations included normal blood work and a normal CXR; a whole-body bone scintigraphy study demonstrated multiple osteoblastic metastases in the skull, ribs, both scapulae, the superior and inferior pubic rami on the left, both femurs and the proximal two-thirds of the right humerus (Fig. 4a).

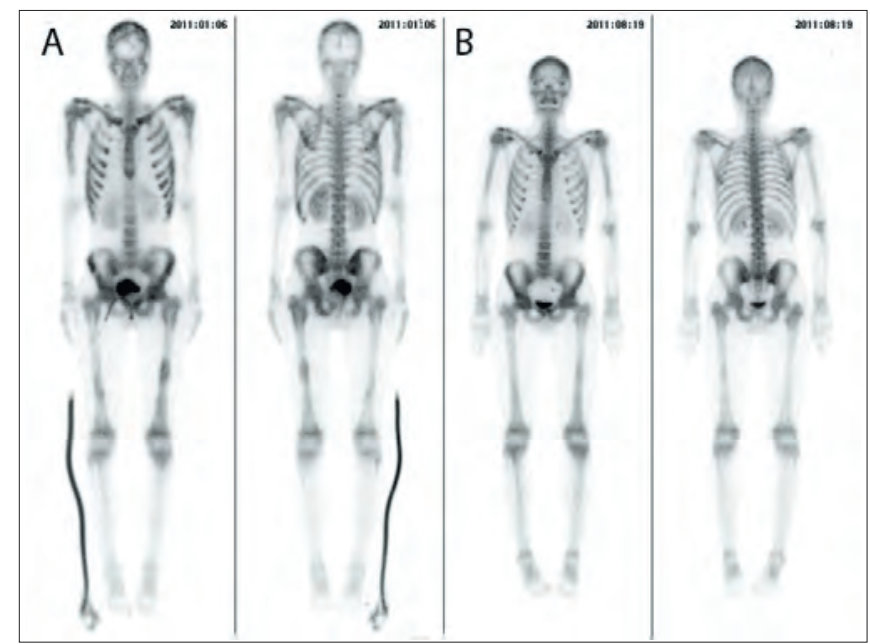

Fig. 4. ${ }^{99 m} T c M D P$ whole-body bone scintigraphy study. (a) Pre-chemotherapy noting multiple osteoblastic metastases in the skull, ribs, both scapulae, the inferior left pubic rami, both femurs and the proximal right humerus. (b) Postchemotherapy study shows no visible metastases, representing a response to chemotherapy.

Focused radiological work-up started with plain film of the pelvis followed by CT of the abdomen and pelvis. Findings on the plain AP pelvis film included a predominantly permeated lytic process that involved the body, superior and inferior rami of the left pubic bone with an associated diffuse interrupted laminated periosteal reaction. Increased surrounding soft-tissue involvement was noted when compared with the contralateral side (Fig. 5). Sequential CT images illustrated the extent of the large heterogeneously enhanced soft-tissue 


\section{CASE SERIES}

tumour that infiltrated the obturator internis muscle and proximal adductor compartment, invaded the pelvic floor on the left, elevated and also infiltrated the bladder base. The included bony window eloquently depicted the aggressive permeated infiltration and associated periosteal reaction (Fig. 6). The additional finding of multiple pulmonary nodules in the included lung basis was consistent with metastatic deposits.

A cystoscopy confirmed tumour in the left bladder neck/dome and was resected for histological investigation. Microscopy revealed

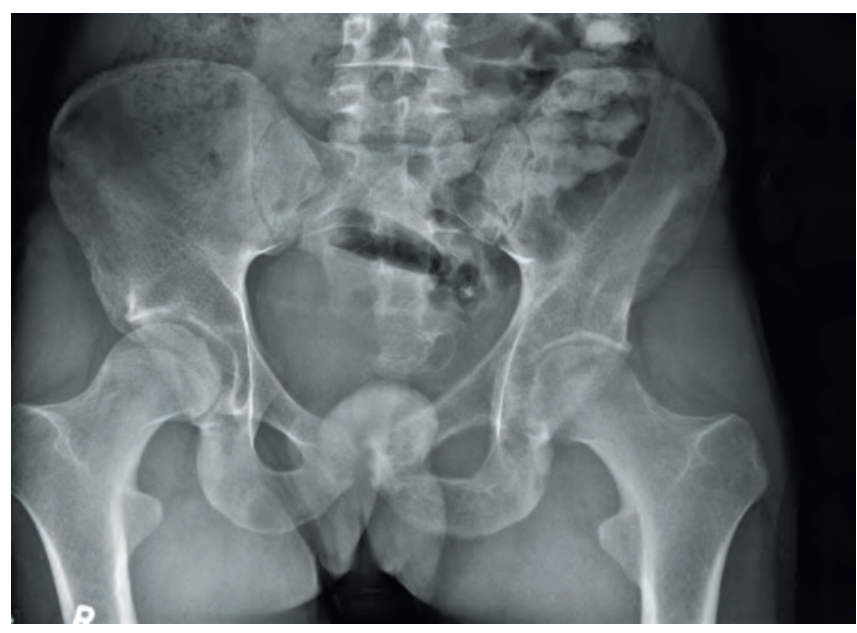

Fig. 5. AP plain film of the pelvis. Note the predominantly permeated lytic process that involves the body, superior and inferior rami of the left pubic bone with an associated diffuse interrupted laminated periosteal reaction.

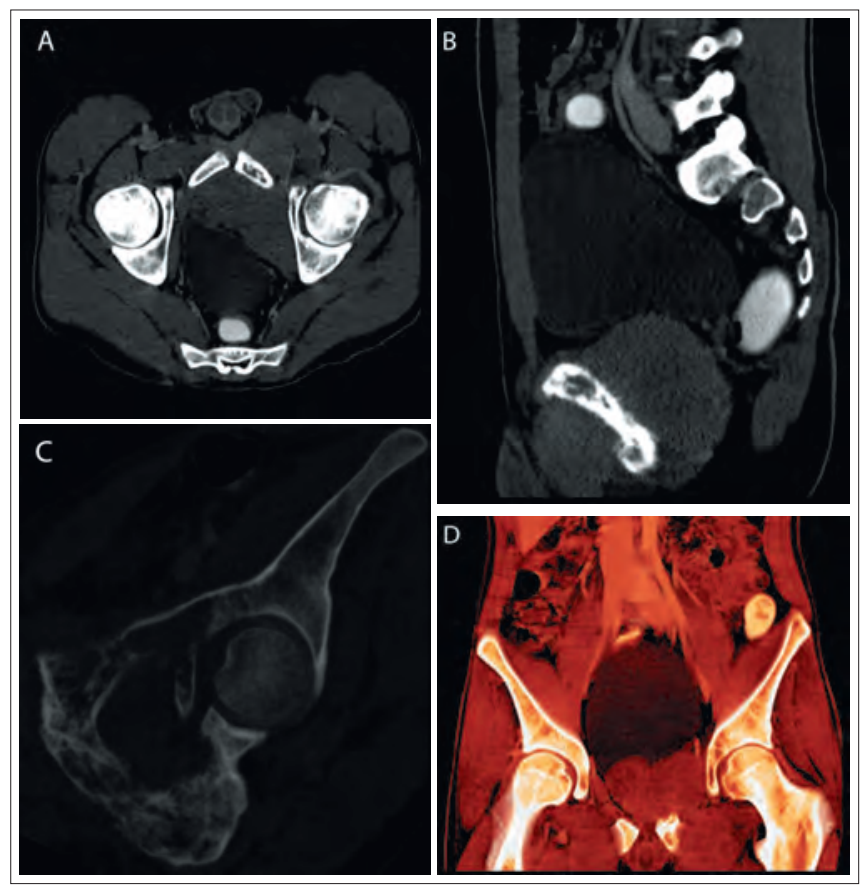

Fig. 6. Sequence of CT images illustrate the extent of the large heterogeneously enhanced soft-tissue tumour that infiltrates the obturator internis muscle and proximal adductor compartment and invades the pelvic floor on the left, also elevated and infiltrating the bladder base. (a) Axial post contrast cut through the pelvis. (b) Sagittal post contrast CT. (c) Oblique coronal CT (bony window depicts the aggressive permeated infiltration and associated periosteal reaction). (d)Volumetric oblique coronal CT. a highly necrotic and malignant small blue cell tumour. Homer Wright-like rosettes were present with high mitotic activity evident. The nuclei contained powdery chromatin, very scanty eosinophilic cytoplasm, inconspicuous nuclei and numerous small vessels. The morphology and immunohistochemistry suggested a possible Ewing's/ PNET tumour, more towards the latter, in view of the rosettes. Some entities were ruled out, such as rhabdomyosarcoma (desmin and myogenin negative), neuroblastoma and lymhoblastic lymphoma (TDT negative). A fluorescence in situ hybridisation test (FISH) was requested for the $t(11 ; 22)$ encoding for EWS-FL11 gene fusion but the outcome was uncertain.

According to the AJCC classification, the patient was staged as a G3T2N0M1, Stage IV Ewing's sarcoma. Palliative systemic chemotherapy with VAIA was offered. He completed 6 cycles with dramatic clinical benefit. Restaging CT was compared with his baseline investigation and the sequential axial post-contrast CT slices through the pelvis confirmed complete resolution of the soft-tissue component as well as the pulmonary nodules. However, residual permeated changes involving the pelvic bones were noted (Figs $7 a$ and $b$ ).

Follow-up scintigraphy study revealed that the previously described metastases were either not visible or far less prominent, most probably
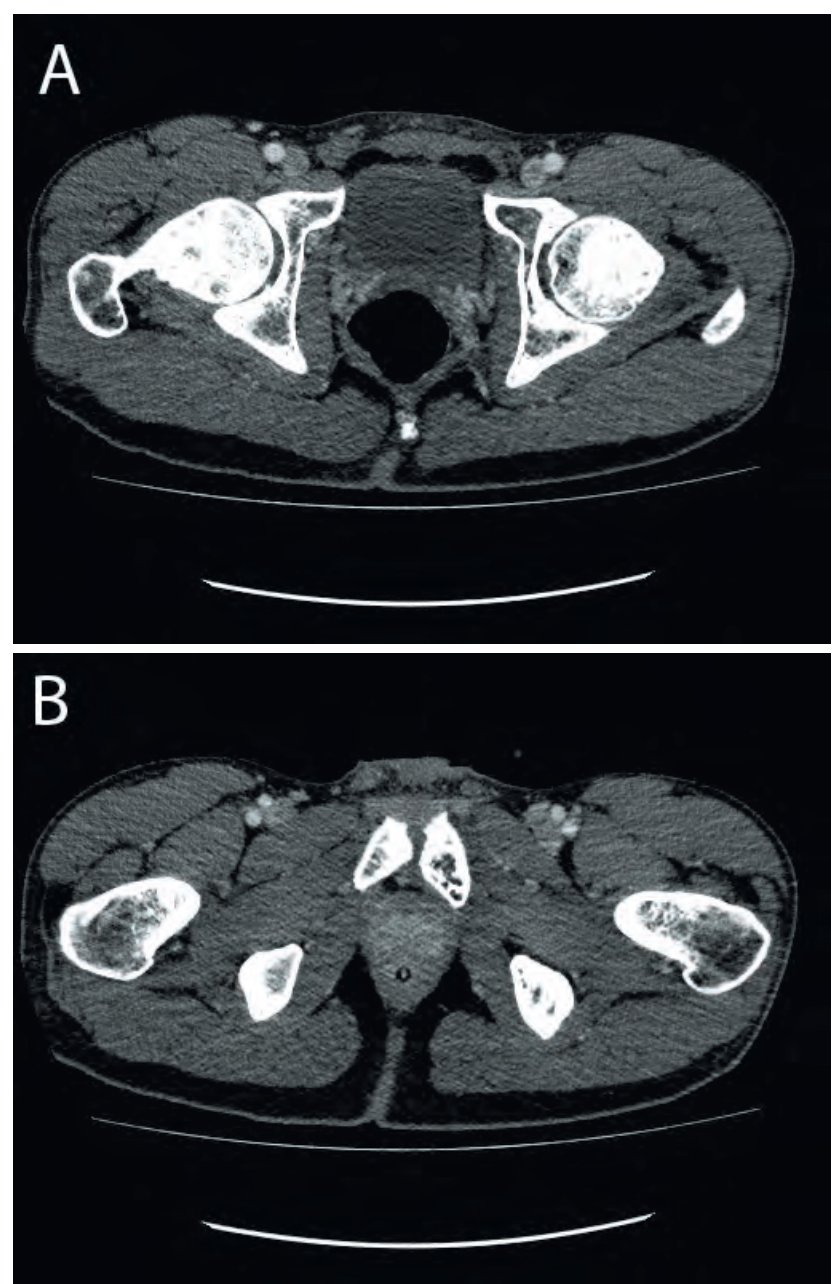

Fig. 7. (a) Pre- and (b) post-chemotherapy. Sequential axial post-contrast $C T$ slices through the pelvis confirm complete resolution of the soft-tissue component. Note the residual permeated changes involving the pelvic bones. 
representing a response to chemotherapy (Fig. 4b). He completed 12 cycles of chemotherapy and is currently in clinico-radiological remission and disease-free for 6 months.

\section{Discussion}

The Ewing's sarcoma family of tumours is an aggressive form of childhood cancer. About $25 \%$ of sufferers will present with metastatic disease, the most common sites for metastases being the lung (50\%), bone (25\%) and bone marrow (20\%). Around 25\% of Ewing's sarcoma arises in soft tissue rather than bone and is referred to as extraskeletal Ewing's sarcoma (ESS); it affects the $10-20$ years age group (range 4 - 25 years), involving the extremities (32\%), paravertebral (15\%), head and neck region $(11 \%)$ and the chest in $11 \%$ of cases. ${ }^{[1,3]}$ However, it has also been reported to arise in the small intestine, kidney, pelvis, skin, oesophagus and paravertebral region or epidural space. Involvement of the spinal epidural space is unusual; only 32 cases have been reported, with a mean age of 19.1 years and a slight predominance in males. The lumbar epidural space (41\%) has been the most commonly reported site. The clinical presentation of spinal ESS relates to compression of the spinal cord, nerve roots or cauda equina, and presents clinically as back pain with or without radicular pain, limb paresis, and bladder and bowel dysfunction. ${ }^{[4]}$ In the literature, primary prostate ESS/PNET is extremely rare, with only 6 cases described. Patient ages ranged from 20 to 30 years. In all cases, the prostate gland was replaced by a large tumour with extraprostatic involvement. The most common CT or MRI finding was a heterogeneously enhanced mass. ${ }^{[5]}$ Clinical differences between ESS and Ewing's sarcoma are few. The long-term prognosis of ESS has not been described but response to chemotherapy has been good and remains palliative for patients with unresectable or metastatic disease. Prognostic indicators in most series include metastatic disease and extraskeletal involvement at presentation. Most patients will succumb to their disease within 2 years. ${ }^{[6]}$

Table 1. Pathological and radiological differential diagnosis

\begin{tabular}{ll}
\hline Small round blue cell tumour & Radiological \\
\hline Non-Hodgkin's lymphoma & Rhabdomyosarcoma \\
Rhabdomyosarcoma & Myosarcoma \\
Small cell lung cancer & Malignant fibrous \\
Merkel cell cancer & histiocytoma (MFH) \\
Haemangiopericytoma & Dedifferentiated liposarcoma \\
Neuroblastoma & Ewing's sarcoma \\
Ewing's sarcoma & \\
Primitive neuro-ectodermal & \\
tumour (PNET) &
\end{tabular}

On imaging, features of Ewing's sarcoma are nonspecific and a radiological differential diagnosis should be considered (Table 1) ${ }^{[5]} \mathrm{CT}$ can show a sharply delineated hypodense soft-tissue mass adjacent to muscle. Enhancement is usually intense and heterogeneous. Hypodense foci are frequent in a large mass and are due to intra-tumour necrosis. ${ }^{[7]}$ On MRI T1-weighted images, the tumour is of low to intermediate signal intensity, and on T2-weighted images high signal intensity with heterogeneous contrast enhancement. Tumour haemorrhage and adjacent bone destruction have been reported. If the mass is located near bone, it might result in cortical erosion and/or a periosteal reaction. ${ }^{[8]} \mathrm{A}$ study by Volker $e t$ al. found whole-body positron emission computer tomography (PET) and conventional imaging were equivalent in detecting primary Ewing's sarcoma, but PET was better at detecting lymph node and bony metastases involvement. ${ }^{[2]}$

In our patients, the radiological CT observed bony changes in the first case were not in keeping with a primary bony neoplasm and thought to be reactive change secondary to the large associated soft-tissue tumor, confirming an ESS. In case 2, the top radiological consideration would rather be a primary Ewing's sarcoma of the pelvic bone with extension into the adjacent soft tissue; radiologically, it is impossible to differentiate from the other members of the Ewing's family. The differential in this case is limited. However, primary bone lymphoma can give similar imaging findings, considering the permeated bony changes and large soft-tissue component.

\section{Conclusion}

ESS arising from soft tissue is rare and has non-specific features on imaging. Both Ewing's sarcoma and ESS are confirmed by features on histological analysis and should be differentiated from other round blue cell tumours and members of the Ewing's family of tumours. In young people who present with soft-tissue tumours, ESS should be considered in the differential diagnosis. Many imaging techniques are available and are most useful in the guidance for biopsy, the evaluation of possible resectability and tumour response to treatment.

1. Grier HE. The Ewing family of tumours: Ewing's sarcoma and primitive neuroectodermal tumours Pediatr Clin North Am 1997;44:991-1004

2. Balamuth NJ, Womer RB. Ewing's sarcoma. Lancet Oncol 2010;11:184-192. [http://dx.doi.org/10.1016/ S1470-2045(09)70286-4]

3. Shirkhoda A, Peuchot M. Extraosseous Ewing's sarcoma computed tomography evaluation before and after chemotherapy. Clinical Imaging 1989;13:142-146

4. Ozturk E, Mutlu H, Sonmez G, et al. Spinal epidural extraskeletal Ewing sarcoma. J Neurorad 2007;34:6367. [http://dx.doi.org/10.4103/2152-7806.96154]

5. Funahasi Y, Yoshino Y. Ewing's sarcoma/primitive neuroectodermal tumour of the prostate. Int $J$ Urol 2009;16:769. [http://dx.doi.org/10.1111/j.1442-2042.2009.02339.x.]

6. Toh KL, Tan PH, Cheng WS. Primary extraskeletal Ewing's sarcoma of the external genitalia. J Urol 1999;162:159-160.

7. Perouli E, Chrysikopoulos H, Vlachos A, et al. Imaging findings in paraspinal extra osseous sarcoma. JBRT-BTR 2006;89:310-312.

8. Ahmad R, Mayol BR, Davids M, Rougraff BT. Extraskeletal Ewing's sarcoma. Cancer 1999; 85:725-731. 